\title{
Contrast-enhanced color Doppler ultrasonography increases diagnostic accuracy for soft tissue tumors
}

\author{
NAOTO OEBISU $^{1}$, MANABU HOSHI $^{1}$, MAKOTO IEGUCHI $^{1}$, JUN TAKADA $^{1}$, \\ TADASHI IWAI $^{1}$, MASAHIKO OHSAWA ${ }^{2}$ and HIROAKI NAKAMURA ${ }^{1}$ \\ Departments of ${ }^{1}$ Orthopedic Surgery and ${ }^{2}$ Diagnostic Pathology, Osaka City University \\ Graduate School of Medicine, Abeno-Ku, Osaka 545-8585, Japan
}

Received June 7, 2014; Accepted July 17, 2014

DOI: $10.3892 / o r .2014 .3378$

\begin{abstract}
Resolution of ultrasonography (US) has undergone marked development. Additionally, a new-generation contrast medium (Sonazoid) used for US is newly available. Contrastenhanced US has been widely used for evaluating several types of cancer. In the present study, we evaluated the ability of color Doppler US (CDUS) and Sonazoid to differentiate between benign and malignant soft tissue tumors. A total of 180 patients ( 87 male, 93 female) were enrolled in the present study. The patient ages ranged from 1 to 91 years (mean $58.1 \pm 20.0$ years). The maximum size, depth, tumor margins, shape, echogenicity and textural pattern were measured on gray-scale images. CDUS was used to evaluate the intratumoral blood flow with and without Sonazoid. Peak systolic flow velocity $(\mathrm{Vp})$, mean flow velocity $(\mathrm{Vm})$, resistivity index (RI) and pulsatility index (PI) of each detected intratumoral artery were automatically calculated with power Doppler US (PDUS). The present study included 118 benign and 62 malignant tumors. Statistical significances were found in size, depth, tumor margin and textural pattern but not in shape or echogenicity on gray-scale images. Before Sonazoid injection, CDUS findings showed 55\% sensitivity, $77 \%$ specificity and $69 \%$ accuracy, whereas contrast-enhanced CDUS showed $87 \%$ sensitivity, $68 \%$ specificity and $74 \%$ accuracy. There were no statistically significant differences between malignant and benign tumors regarding the mean $\mathrm{Vp}, \mathrm{Vm}, \mathrm{RI}$ and PI values determined on PDUS. In conclusion, contrast-enhanced CDUS proved to be a reliable diagnostic tool for detecting malignant potential in soft tissue tumors.
\end{abstract}

Correspondence to: Dr Naoto Oebisu, Department of Orthopedic Surgery, Osaka City University Graduate School of Medicine, 1-4-3 Asahi-Machi, Abeno-Ku, Osaka 545-8585, Japan

E-mail: evis@med.osaka-cu.ac.jp

Key words: contrast-enhanced ultrasonography, soft tissue tumor, vascularity, sonazoid

\section{Introduction}

Soft tissue tumors are not rare findings in daily orthopedic practice. Several imaging modalities have been applied to assess these tumors, including plain radiography, nuclear medicine, computed tomography (CT), magnetic resonance imaging (MRI), ultrasonography (US), angiography and positron emission tomography. Most general practitioners, however, find it difficult to distinguish benign from malignant lesions (1). Although MRI and CT are the most common modalities for evaluating soft tissue masses, many patients have difficulty undergoing these examinations. Additionally, their cost may be prohibitive.

Clinical radiologists and orthopedic oncologists are urgently seeking a tool that can identify malignant potential in these soft tissue tumors. Among the various imaging modalities, US is most often available clinically. It also has the advantages of being simple, easy and inexpensive, and it can offer results in real-time. Conversely, US has not been found to be reliable for examining soft tissue tumors as little information is available about differentiating benign from malignant lesions by US (2).

In recent years, the resolution of US has undergone marked development. Concurrently, a new-generation contrast medium for US has been approved for use. Several previous studies confirmed that intratumoral blood flow was a useful factor for differentiating benign and malignant tumors (2-4). It is possible that combining US with contrast medium can increase the accuracy of detecting malignant potential in soft tissue tumors. The aim of the present study was to elucidate the usefulness of contrast-enhanced color Doppler US (CDUS) in the preoperative differential diagnosis of benign and malignant soft tissue tumors.

\section{Materials and methods}

Between January 2010 and December 2013, a total of 180 patients ( 87 male, 93 female) were enrolled in the present study. The patient ages ranged from 1 to 91 years (mean 58.1 \pm 20.0 years). The patients were treated at Osaka City University Hospital in Osaka, Japan. The institutional Ethics Review Board of Osaka City University Graduate School of Medicine approved the protocol of the present study. 

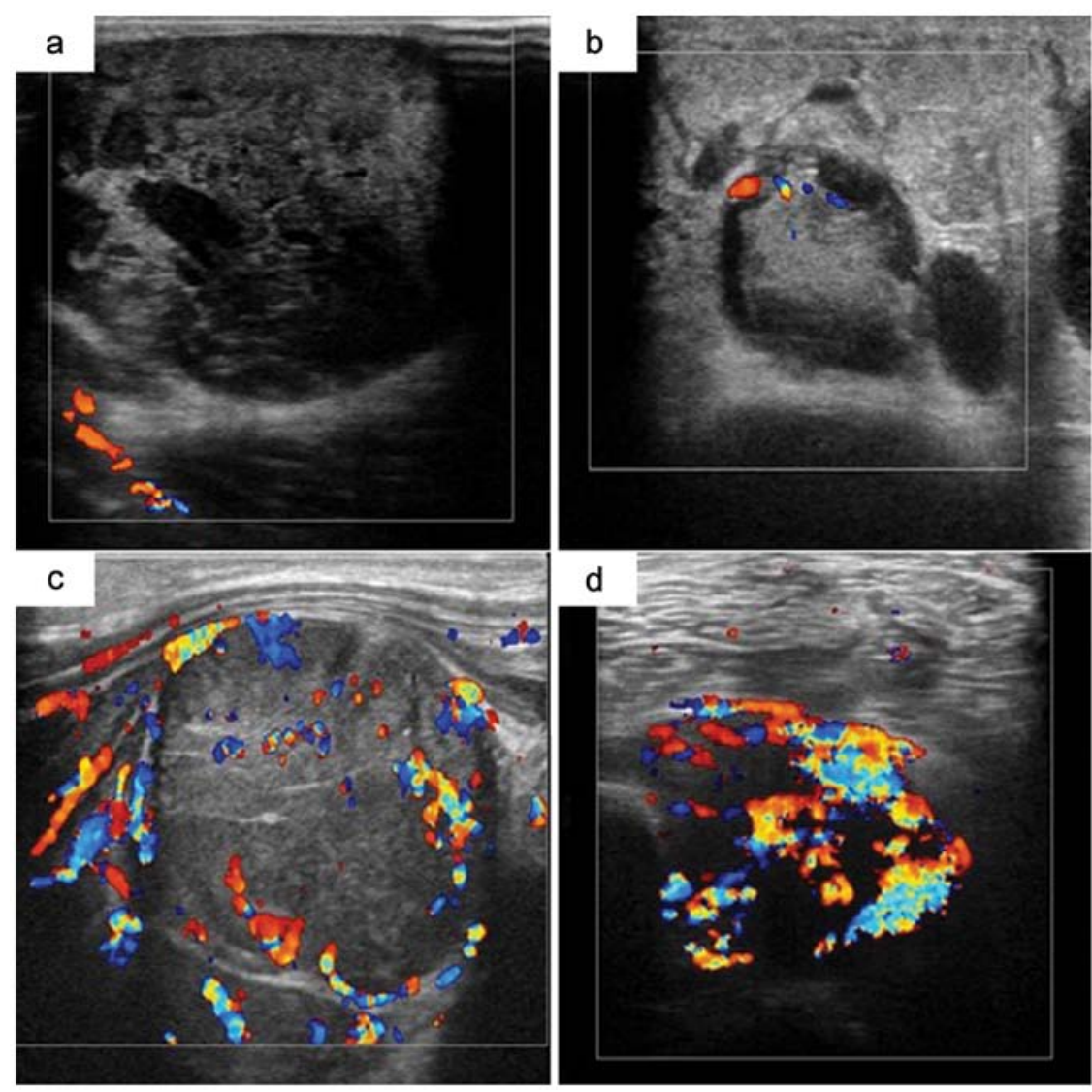

Figure 1. Color Doppler ultrasonography (CDUS) of intratumoral blood flow, according to the classification of Giovagnorio et al (3). (a) Grade I epidermal cyst from the right back of a 71-year old man. (b) Grade II ganglion cyst from the left leg of a 73-year old woman. (c) Grade III malignant fibrous histiocytoma from the left thigh of a 77-year old man. (d) Grade IV pleomorphic liposarcoma from the right thigh of a 65 -year old woman.

All the patients who presented with soft tissue masses were screened by US at the first or second visit to our institution. Histologic confirmation is often important for the diagnosis. Thus, ultimately, all these patients underwent biopsy or tumor resection. A pathologist with expertise in sarcoma pathology examined the specimens according to standard criteria for bone and soft tissue sarcoma subtyping based on the World Health Organization classification system (5). All the patients provided written permission to use their samples.

We used the HI VISION Avius US apparatus (Hitachi-Aloka Medical, Tokyo, Japan). The linear array transducers had multifrequencies of 5.0 MHz. Sonazoid (Daiichi Sankyo, Tokyo, Japan), a second-generation contrast medium designed for US, was used to assess the vascularity of the tumors. Sonazoid was injected intravenously as a $0.5-\mathrm{ml}$ bolus followed by a $10-\mathrm{ml}$ normal saline flush using a 22-gauge peripheral intravenous cannula. US images were recorded on a hard disk starting $40 \mathrm{sec}$ after the injection and continuing for up to $2 \mathrm{~min}$.

Before injecting the Sonazoid, gray-scale US was used to measure the maximum size, depth of the soft tissue masses, tumor margins, shape (round, ovoid, lobulated), echogenicity (hyperintense, isointense, hypointense) and textural pattern (homogeneous or heterogeneous). US also identified its anatomical location. Tumor margins were assessed as well-defined (clear-cut and thin capsule-like) or ill-defined (uncertain margin with respect to adjacent normal tissue).

CDUS was used to evaluate the blood flow with and without Sonazoid. Based on Giovagnorio et al criteria (3), the CDUS
Table I. Clinical information of patient characteristics.

\begin{tabular}{lcc}
\hline Variable & Benign (No.) & Malignant (No.) \\
\hline & 118 & 62 \\
Median age & $54(1-84)$ & $64(12-91)$ \\
Gender & & \\
Male & 50 & 37 \\
Female & 68 & 25 \\
Location of lesion & & \\
Head or neck & 1 & 0 \\
Trunk & 13 & 14 \\
Arm or elbow & 10 & 8 \\
Forearm & 4 & 1 \\
Hand & 18 & 0 \\
Thigh & 31 & 31 \\
Knee of leg & 25 & 8 \\
Ankle of foot & 16 & 0 \\
\hline
\end{tabular}

grades were: I (avascular); II (hypovascular with a single vascular pole); III (hypervascular with multiple peripheral poles); or IV (hypervascular with internal vessels). Grade I and II masses were categorized as benign, whereas grades III and IV masses were classified as malignant (Fig. 1). 
Table II. Histological diagnosis of each patient.

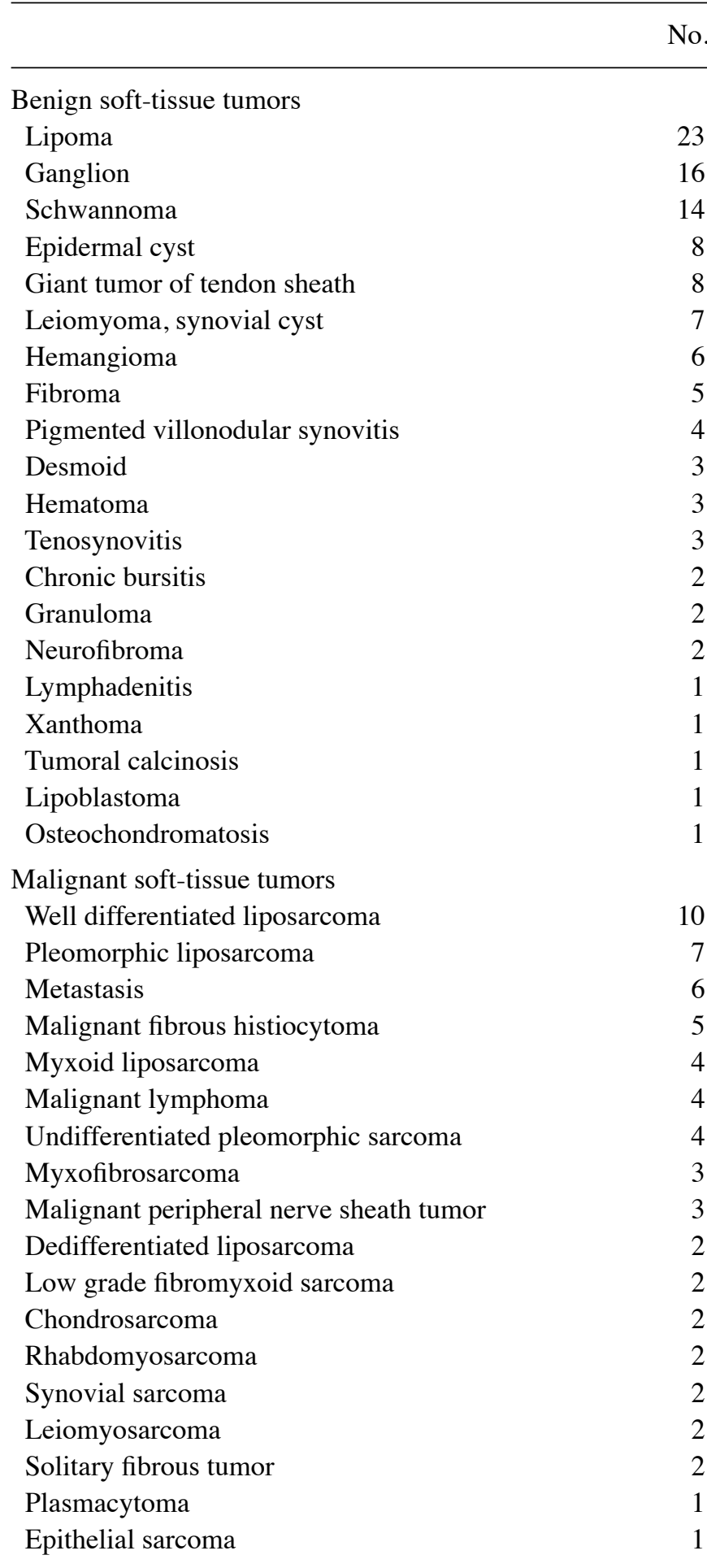

We assessed the vascular velocity inside the tumor by measuring the main artery from 1 to 3 vessels and the mean value was calculated. The peak systolic flow velocity $(\mathrm{Vp})$, mean flow velocity $(\mathrm{Vm})$, resistivity index (RI) and pulsatility index (PI) of each detected intratumoral artery were assessed with power Doppler US (PDUS). The RI was defined by the following equation: (peak systolic velocity - end-diastolic velocity)/peak systolic velocity. The PI was defined as follows: (peak systolic velocity - end-diastolic velocity)/time average velocity.
Table III. Comparison with gray-scale of benign and malignant tumors.

\begin{tabular}{lccc}
\hline Variable & Benign & Malignant & P-value \\
\hline Mean size (mm) & $39.6 \pm 17.3$ & $78.6 \pm 31.4$ & 0.0001 \\
Mean depth (mm) & $5.7 \pm 2.9$ & $9.0 \pm 3.2$ & 0.0012 \\
Shape & & & 0.71 \\
Round & 59 & 26 & \\
Ovoid & 26 & 22 & \\
Lobulated & 33 & 14 & \\
Echogenicity & & & 0.61 \\
Hyper & 38 & 21 & \\
Hypo & 63 & 27 & \\
Iso & 17 & 14 & \\
Textural pattern & & & \\
Homo & 73 & 23 & \\
Hetero & 45 & 39 & \\
Tumor margin & & & \\
Well-defined & 106 & 42 & \\
Ill-defined & 12 & 200017 \\
\hline
\end{tabular}

Statistical analysis. Quantitative data are presented as the means \pm standard deviation. The Mann-Whitney U, Fisher's exact and $\chi^{2}$ tests were used for unpaired comparisons between the quantitative parameters. Statistical analysis was performed using Excel statistics software for Windows (version 2012; SSRI Co., Ltd., Tokyo, Japan). A value of $\mathrm{p}<0.05$ was considered to indicate a statistically significant difference.

\section{Results}

Patient characteristics. A total of 118 benign and 62 malignant tumors were included in the present study (Table I). Benign soft tissue masses were located in the neck $(n=1)$, trunk $(n=13)$, upper arm $(n=10)$, forearm $(n=4)$, hand $(n=18)$, thigh $(n=31)$, lower thigh $(n=25)$ and foot $(n=16)$. Malignant soft tissue masses were located in the trunk $(n=14)$, upper $\operatorname{arm}(n=8)$, forearm $(n=1)$, thigh $(n=31)$ and lower thigh $(n=8)$. Table II shows the histologic diagnosis of benign and malignant tumors.

Comparison of gray-scale US images. The mean sizes of benign and malignant soft tissue tumors were calculated to be $39.8 \pm 17.3$ and $78.6 \pm 31.4 \mathrm{~mm}$, respectively (Table III). The mean depths were $5.7 \pm 2.9$ and $9.0 \pm 3.2 \mathrm{~mm}$, respectively. The differences in these two factors (size and depth) were statistically significant $(\mathrm{p}<0.001)$. The margins of benign soft tissue tumors were ill-defined in 12 of $118(10.2 \%)$ tumors, whereas those of malignant tumors were ill-defined in 20 of 62 (32.3\%) tumors $(\mathrm{p}<0.001)$. Regarding the textural pattern, the benign soft tissue tumors were homogeneous in 73 of 118 (61.9\%), whereas the malignant tumors were homogeneous in 23 of 62 $(37.1 \%)(p=0.0017)$. The differences in shape and echogenicity were not statistically significant. 
Table IV. Comparison with CDUS findings of benign and malignant tumors.

\begin{tabular}{|c|c|c|c|c|c|}
\hline Variable & Benign (No.) & Malignant (No.) & Sensitivity (\%) & Specificity (\%) & Accuracy $(\%)$ \\
\hline \multicolumn{6}{|c|}{ CDUS grade without CM } \\
\hline Grade I, II & 91 & 28 & 54.8 & 77.1 & 69.4 \\
\hline Grade III, IV & 27 & 34 & & & \\
\hline \multicolumn{6}{|c|}{ CDUS grade with CM } \\
\hline Grade I, II & 48 & 5 & 86.8 & 67.6 & 74.3 \\
\hline Grade III, IV & 23 & 33 & & & \\
\hline
\end{tabular}

CDUS, color Doppler ultrasonography; CM, contrast medium.

Table V. Comparison with PDUS findings of benign and malignant tumors.

\begin{tabular}{lrcc}
\hline Variable & Benign & Malignant & P-value \\
\hline Mean Vp $(\mathrm{cm} / \mathrm{sec})$ & 18.1 & 16.5 & 0.45 \\
Mean Vm $(\mathrm{cm} / \mathrm{sec})$ & 6.5 & 8.1 & 0.19 \\
Mean PI & 4.67 & 3.46 & 0.36 \\
Mean RI & 0.95 & 1.14 & 0.47
\end{tabular}

PDUS, power Doppler ultrasonography; Vp, peak systolic flow velocity; Vm, mean flow velocity; RI, resistivity index; PI, pulsatility index.

Comparison of CDUS findings. In total, 109 patients received intravenous injections of Sonazoid. Before Sonazoid injection, the tumor grades on CDUS were, respectively, I, II, III or IV in 52, 39, 14 and 13 benign tumors and in 7, 21, 12 and 21 malignant tumors. After Sonazoid injection, the grades were I, II, III and IV, respectively, in 23, 25, 7 and 16 benign tumors and in 2,3,11 and 22 malignant tumors. The probability of malignancy on CDUS findings before Sonazoid injection were $55 \%$ sensitivity, $77 \%$ specificity, $76 \%$ negative predictive value (NPV), 56\% positive predictive value (PPV) and 69\% accuracy. Following administration of Sonazoid, these values increased to $86 \%$ sensitivity, $68 \%$ specificity, $90 \%$ NPP, 59\% PPV and $74 \%$ accuracy (Table IV).

Comparison of PDUS findings. PDUS analyses of benign tumors showed that the mean $\mathrm{Vp}, \mathrm{Vm}, \mathrm{RI}$ and PI values were $18.1 \mathrm{~cm} / \mathrm{sec}(1.5-43.0), 6.5 \mathrm{~cm} / \mathrm{sec}(0.4-20.2), 0.95(0-1.7)$ and 4.67 (0.03-49.5), respectively (Table V). In the malignant groups, the corresponding values were $16.5 \mathrm{~cm} / \mathrm{s}(4.5-41.0)$, $8.1 \mathrm{~cm} / \mathrm{s}(0.2-33.1), 1.14(0.14-10.8)$ and $3.46(0.16-18.7)$, respectively (Table V). The differences between the mean values of each parameter for the malignant and benign tumor groups were not statistically significant.

\section{Discussion}

Soft tissue tumors are commonly found in the everyday practice of general surgeons, including orthopedic surgeons. The incidence of malignant soft tissue tumors is low, accounting
Table VI. Previous reports of US for soft-tissue tumors.

\begin{tabular}{llr}
\hline Author (Refs.) & Patient & \multicolumn{1}{c}{ Result (\%) } \\
\hline Lagalla et al (2) & 46 Soft-tissue & US: Sensitivity (75) \\
& masses & Specificity (50) \\
& & Accuracy (61) \\
& & CDUS: Sensitivity (85) \\
et al (3) & & Specificity (92) \\
Belli et al (25) & 71 Nodules & Accuracy (89) \\
& & CDUS: Sensitivity (90) \\
& 20 Menign & Specificity (100) \\
& tumors & US: Sensitivity (60) \\
& & Specificity (55) \\
& & Accuracy (57) \\
& & CDUS: Sensitivity (85) \\
Present study & 118 Benign & Specificity (88) \\
& 62 Malignant & Accuracy (87) \\
& & CDUS: Sensitivity (55) \\
& & Specificity (77) \\
& & Accuracy (69) \\
& & CEUS: Sensitivity (87) \\
& & Accuracy (74) \\
& &
\end{tabular}

US, ultrasonography; CDUS, color Doppler US; CEUS, contrast enhanced US.

for $<1 \%$ of all neoplasms. In contrast, benign soft tissue tumors occur rather frequently (6). In the USA, the annual incidence of soft tissue tumors was calculated at $\sim 3$ cases $/ 1,000$ people, with $\sim 0.69 \%$ of them considered malignant (7). Although the precise number of malignant soft tissue tumors in Japan is not known, it has been estimated at around 2 cases/10,000 people according to the Soft Tissue Tumor Registry in Japan (JOA Musculoskeletal Tumor Committee, 2008).

Several imaging modalities have been used to assess soft tissue tumors, with CT and MRI playing a key role. Several authors (8-10) noted that only MRI and CT can assess the possible components of the tumors and can show their anatomic location and extent. However, with the exception of lipomatous 

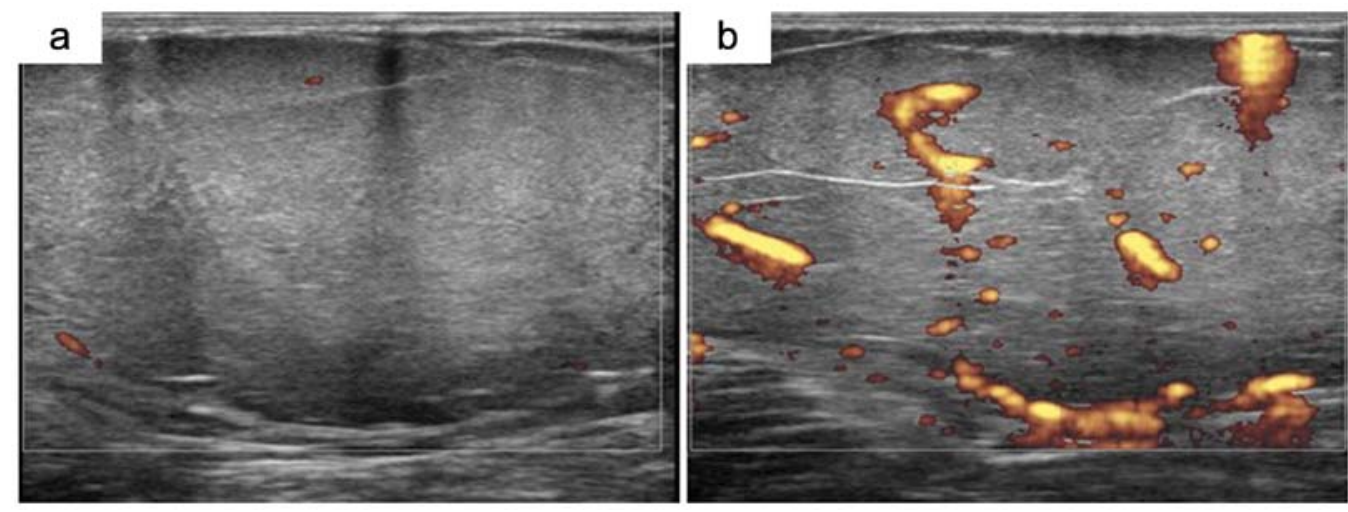

Figure 2. Well-differentiated liposarcomas from the right thigh of a 36-year old woman. (a) CDUS image (grade II) before injection with Sonazoid. (b) CDUS image (grade IV) after administration of Sonazoid. CDUS, color Doppler ultrasonography.

tumors, schwannomas and myositis ossificans, they do not allow a definitive histological diagnosis (11-13). Nevertheless, MRI and CT can generally provide important information for a preoperative diagnosis and for planning surgery (14).

Various medical specialists, including dermatologists, plastic and general surgeons, treat soft tissue tumors .Unplanned resections can engender serious problems. The unplanned resection of a sarcoma is defined as 'an excisional biopsy or unplanned resection of lesion without the benefits of preoperative imaging and without the benefits of preoperative imaging and without regard for the necessity of removing the lesion with a margin of normal tissue' (15). Occasionally, surgery is undertaken without sufficient preoperative images (16). Since MRI and CT are not always available locally, the patient cannot be examined immediately. Additionally, MRI is costly and radiologists are not always equipped to review the images. Therefore, an easy universal screening modality to detect soft tissue masses is desirable.

We focused on US as a powerful candidate for screening patients for soft tissue tumors. US is non-invasive, has a low cost, and is widely available compared with CT and MRI. It is also possible to perform US in an ambulatory practice. To date, however, little evidence-based information has been available concerning the use of US to evaluate soft tissue tumors. Hence, we decided to test the usefulness of US with the latest material available to determine if it can contribute to distinguishing between benign and malignant soft tissue tumors.

Several previous reports have introduced the feasibility of US in differentiating benign and malignant soft tissue tumors $(2,17-21)$. In the present study, the sizes, depths, tumor margins and textural patterns were significantly different between benign and malignant soft tissue tumors on gray-scale US images. Lange et al (20) noted that soft tissue masses with an ill-defined appearance on US may be assumed to be benign. In the present study, most tumors had a well-defined appearance, whereas an ill-defined appearance was considered malignant; quite different from the report of Lange et al (20). Advances in image resolution enabled this distinction. Chiou et al (21) reported that the tumor margin, shape and size seemed to be significant indicators for differentiating benign from malignant soft tissue tumors, which is consistent with the results of the present study. However, shape and echogenicity of tumors were not significantly different.
The evaluation of intratumoral vascularity is an important clue for distinguishing benign from malignant lesions (19,22-24). Malignant tumors show an increased number of vessels (23). Ma et al (24) noted that intratumoral enhancement patterns of malignant and benign masses differ due to differences in their vascular architecture. CDUS is able to evaluate intratumoral blood flow in real-time. Belli et al (25) demonstrated that conventional sonography was not reliable for diagnosing malignancy, whereas color imaging and power Doppler imaging were quite useful. Lagalla et al (2) stated that malignant potential was indicated in the presence of three or more vascular signals and tortuous and irregular internal vessels seen by CDUS. In contrast, Chiou et al (21) denied the usefulness of CDUS features for differentiating benign from malignant soft tissue tumors. Giovagnorio et al (3) classified tumors based on four vascular patterns. Among them, hypervascular tumors with multiple peripheral poles (type III) and hypervascular tumors with internal vessels (type IV) were assumed to be malignant. We used their classification in the present study to differentiate benign and malignant tumors on CDUS. The CDUS results, however, demonstrated lower sensitivity (54.8\%) and accuracy (69.4\%) than were reported in previous studies (Table VI). We concluded that the CDUS images were not good enough to screen for malignancy in soft tissue tumors.

Since contrast medium may cause fine vascular structures to appear more vividly, it was expected that contrast-enhanced CDUS may contribute to clearer differentiation of soft tissue tumors. This method has been widely used in several fields, including those that address hepatic, breast and prostate diseases (26-28). Chiou et al (29) described that, for soft tissue tumors, contrast-enhanced US was markedly better than noncontrast-enhanced images for detecting vessels in most tumors.

Sonazoid is a second-generation contrast agent designed for US use and has been approved in recent years. It consists of micro-bubbles filled with chemically stable, poorly soluble gas. The use of Sonazoid makes it possible to evaluate intratumoral blood flow for a longer time and in more detail than when using the previous-generation medium Levovist. We hypothesized that we could distinguish between benign and malignant tumors more clearly with Sonazoid than without it.

In the present study, contrast-enhanced CDUS demonstrated higher sensitivity (86.8\%) and accuracy (74.3\%) than 
did the CDUS-alone findings. Contrast-enhanced CDUS images enabled the diagnoses of some low-grade malignant tumors, well-differentiated liposarcomas and low-grade fibromyxoid sarcomas. In 10 patients with well-differentiated liposarcomas, CDUS images suggested vascular and hypovascular components in all cases. After administration of contrast medium, CDUS displayed hypervascularity in 7 of the 10 cases (Fig. 2). Both cases of low-grade fibromyxoid sarcoma also showed hypovascularity on CDUS-alone images, whereas after adding contrast medium, CDUS revealed hypervascularity in both cases.

All orthopedic oncologists possibly know from experience that during intralesional resection of a tumor the amount of intraoperative bleeding is greater with a malignant tumor than with a benign tumor. Hence, we hypothesized that the blood velocity is greater inside a malignant tumor than in a benign tumor. Additionally, malignant tumors form tortuous, deformed and displaced vascular structures that may be highly unstable and immature. They are characterized by a high proliferation of endothelial cells, hyperpermeability and chaotic blood flow (30). We surmised that the PI and RI were also enhanced in malignant tumors and thus could indicate a diagnosis of malignancy. Regarding PDUS findings in soft tissue tumors, Belli et al (25) noted that vessel characteristic analysis using PDUS had $85 \%$ sensitivity and $88 \%$ specificity. Bodner et al (31) discovered that the RI ratio was an indicator of malignancy. The present study, however, found no statistically significant differences in mean Vp, Vm, RI and PI values. Some benign tumors (such as schwannoma, hemangioma, lymphadenitis) show hypervascularity, with Vp, Vm, RI and PI values of these tumors being close to those for malignant tumors. In this case, our results were not consistent with those previously reported $(25,31)$.

The present study has several limitations. First, the sample size is small, and there were various histologic diagnoses. Second, tumor vascularity varies during different phases of tumor growth. When tumor growth progresses, areas of hypoxia and necrosis may appear. We failed to estimate US findings in connection with necrosis confirmed by pathology. Third, CDUS displayed false-positive and false-negative findings for some tumors. As already mentioned, well-differentiated liposarcomas and low-grade fibromyxoid sarcomas were typically false-negative tumors. Conversely, schwannoma with representative histology showed a false-positive finding. In total, 9 of 14 (64\%) patients with schwannoma displayed hypervascularity on the images. To solve the problem of false-positive and false-negative findings, we should focus on these specific tumors and determine the specific findings for each tumor. Since this histology is rare, however, it would be difficult to accumulate these data.

In conclusion, tumor size, depth, textural pattern and tumor margin proved to be positive parameters on gray-scale US images for differentiating benign from malignant tumors. From the standpoint of vascularity within the tumor, CDUS showed lower sensitivity (54.8\%) and lower accuracy (69.4\%). Contrast medium administration enhanced sensitivity and accuracy up to 86.8 and $74.4 \%$, respectively. PDUS provided no useful information. Contrast-enhanced CDUS proved to be a reliable diagnostic tool with which to screen for malignant potential in soft tissue tumors.

\section{References}

1. Kransdorf MJ and Murphey MD: Radiologic evaluation of softtissue masses: a current perspective. AJR Am J Roentgenol 175: 575-587, 2000.

2. Lagalla R, Iovane A, Caruso G, Lo Bello M and Derchi LE: Color Doppler ultrasonography of soft-tissue masses. Acta Radiol 39: 421-426, 1998.

3. Giovagnorio F, Andreoli C and De Cicco ML: Color Doppler sonography of focal lesions of the skin and subcutaneous tissue. J Ultrasound Med 18: 89-93, 1999.

4. Widmann G, Riedl A, Schoepf D, Glodny B, Peer S and Gruber H: State-of-the-art HR-US imaging findings of the most frequent musculoskeletal soft-tissue tumors. Skeletal Radiol 38: 637-649, 2009.

5. Jo VY and Fletcher CD: WHO classification of soft tissue tumours: an update based on the 2013 (4th) edition. Pathology 46: 95-104, 2014.

6. Simon MA and Finn HA: Diagnostic strategy for bone and softtissue tumors. J Bone Joint Surg Am 75: 622-631, 1999.

7. Siegel R, Naishadham D and Jemal A: Cancer statistics, 2013. CA Cancer J Clin 63: 11-30, 2013.

8. Drapé JL: Advances in magnetic resonance imaging of musculoskeletal tumours. Orthop Traumatol Surg Res 99 (Suppl 1): S115-S123, 2013.

9. Landa $\mathbf{J}$ and Schwartz LH: Contemporary imaging in sarcoma. Oncologist 14: 1021-1038, 2009.

10. Iwamoto Y: Diagnosis and treatment of soft tissue tumors. J Orthop Sci 4: 54-65, 1999.

11. Simon MA and Biermann JS: Biopsy of bone and soft-tissue lesions. J Bone Joint Surg Am 75: 616-621, 1993.

12. Stacy GS and Dixon LB: Pitfalls in MR image interpretation prompting referrals to an orthopedic oncology clinic. Radiographics 27: 805-828, 2007.

13. Kawaguchi N, Matsumoto $S$ and Manabe J: Clinical diagnosis of soft tissue tumors. J Orthop Sci 3: 225-238, 1998.

14. ESMO/European Sarcoma Network Working Group: Soft tissue and visceral sarcomas: ESMO Clinical Practice Guidelines for diagnosis, treatment and follow-up. Ann Oncol 23 (Suppl 7): vii92-vii99, 2012.

15. Noria S, Davis A, Kandel R, Levesque J, O'Sullivan B, Wunder J and Bell R: Residual disease following unplanned excision of soft-tissue sarcoma of an extremity. J Bone Joint Surg Am 78: 650-655, 1996.

16. Hoshi M, Ieguchi M, Takami M, Aono M, Taguchi S, Kuroda T and Takaoka K: Clinical problems after initial unplanned resection of sarcoma. Jpn J Clin Oncol 38: 701-709, 2008.

17. Jin W, Kim GY, Park SY, Chun YS, Rhyu KH, Park JS and Ryu KN: The spectrum of vascularized superficial soft-tissue tumors on sonography with a histopathologic correlation: part 2, malignant tumors and their look-alikes. AJR Am J Roentgenol 195: 446-453, 2010.

18. Braunstein EM, Silver TM, Martel $\mathrm{W}$ and Jaffe M: Ultrasonographic diagnosis of extremity masses. Skeletal Radiol 6: 157-163, 1981.

19. Adler RS, Bell DS, Bamber JC, Moskovic E and Thomas JM: Evaluation of soft-tissue masses using segmented color Doppler velocity images: preliminary observations. AJR Am J Roentgenol 172: 781-788, 1999.

20. Lange TA, Austin CW, Seibert JJ, Angtuaco TL and Yandow DR: Ultrasound imaging as a screening study for malignant softtissue tumors. J Bone Joint Surg Am 69: 100-105, 1987.

21. Chiou HJ, Chou YH, Chiu SY, Wang HK, Chen WM, Chen TH and Chang CY: Differentiation of benign and malignant superficial soft-tissue masses using grayscale and color doppler ultrasonography. J Chin Med Assoc 72: 307-315, 2009.

22. Jin W, Kim GY, Park SY, Chun YS, Nam DH, Park JS and Ryu KN: The spectrum of vascularized superficial soft-tissue tumors on sonography with a histopathologic correlation: part 1 , benign tumors. AJR Am J Roentgenol 195: 439-445, 2010.

23. Verstraete KL, Van der Woude HJ, Hogendoorn PC, De-Deene Y, Kunnen M and Bloem JL: Dynamic contrast-enhanced MR imaging of musculoskeletal tumors: basic principles and clinical applications. J Magn Reson Imaging 6: 311-321, 1996.

24. Ma LD, Frassica FJ, McCarthy EF, Bluemke DA and Zerhouni EA: Benign and malignant musculoskeletal masses: MR imaging differentiation with rim-to-center differential enhancement ratios. Radiology 202: 739-744, 1997. 
25. Belli P, Costantini M, Mirk P, Maresca G, Priolo F and Marano P: Role of color Doppler sonography in the assessment of musculoskeletal soft tissue masses. J Ultrasound Med 19: 823-830, 2000.

26. Omoto K, Matsunaga $\mathrm{H}$, Take N, et al: Sentinel node detection method using contrast-enhanced ultrasonography with sonazoid in breast cancer: preliminary clinical study. Ultrasound Med Biol 35: 1249-1256, 2009.

27. Uemura H, Sano F, Nomiya A, et al: Usefulness of perflubutane microbubble-enhanced ultrasound in imaging and detection of prostate cancer: phase II multicenter clinical trial. World J Urol 31: 1123-1128, 2013.

28. Goto E, Masuzaki R, Tateishi R, et al: Value of post-vascular phase (Kupffer imaging) by contrast-enhanced ultrasonography using Sonazoid in the detection of hepatocellular carcinoma. J Gastroenterol 47: 477-485, 2012.
29. Chiou HJ, Chou YH, Chen WM, Chen W, Wang HK and Chang CY: Soft-tissue tumor differentiation using 3D power Doppler ultrasonography with echo-contrast medium injection. J Chin Med Assoc 73: 628-633, 2010.

30. Metheny-Barlow LJ and Li LY: The enigmatic role of angiopoietin-1 in tumor angiogenesis. Cell Res 13: 309-317, 2003.

31. Bodner G, Schocke MF, Rachbauer F, Seppi K, Peer S, Fierlinger A, Sununu T and Jaschke WR: Differentiation of malignant and benign musculoskeletal tumors: combined color and power Doppler US and spectral wave analysis. Radiology 223: 410-416, 2002. 